\title{
A cueing technique in choice reaction time
}

\author{
DAVID LaBERGE, ${ }^{2}$ PETER VAN GELDER, AND JOHN YELLOTT, JR. \\ UNIVERSITY OF MINNESOTA
}

\begin{abstract}
Much of the fluctuation in choice RT data is assumed to arise from variations in perceptual and response biases correlated with sequential patterns of trial events. Inserting a predictive cue prior to the stimulus on each trial apparently induces a strong bias not only toward that particular stimulus, but also toward its modality and toward the associated response. Trial-to-trial sequential effects under cued conditions were markedly smaller than those obtained in a noncued control condition.
\end{abstract}

This paper describes a modification of the standard choice reaction time (RT) task that was designed to permit better trial-by-trial control over S's perceptual and response biases. A standard choice RT experiment consists of a sequence of independent trials in which the choice stimuli $s_{1}, s_{2}, \cdots, s_{N}$ have fixed probabilities $\pi_{1}, \pi_{2}, \cdots, \pi_{N}$ of being presented on any trial, regardless of events occurring earlier in the sequence. Despite this independence between successive stimuli, it is commonly found that S's response to any given presentation of Stimulus $s_{i}$ is significantly influenced by the particular sequence of stimuli presented on immediately preceding trials. These sequential effects can be quite substantial; in one condition of a study reported by Bertelson and Renkin (1966), mean latency was $85 \mathrm{msec}$ faster on trials involving a repetition of the preceding stimulus than on trials involving nonrepetitions. Similar positive recency effects have been found in other experiments by Bertelson (1961, 1963), and by Falmange (1965). Other studies, however, have discovered negative recency effects (faster responding to non repetitions; Williams, 1966), and recently Laming (1969) has reported an experiment in which both positive and negative recency effects were obtained: RT was slowest on trials that followed exactly one repetition of the current stimulus, faster following a stimulus different from the current one, and faster still after a run of two or more repetitions of the current stimulus. Apparently the direction of sequential effects and their exact form depend on specific experimental parameters (e.g., the intertrial interval), and on idiosyncratic

Fig. 1. Schematic representation of events within a cued trial $(A)$ and an uncued trial (B). biases carried over from earlier experimental sessions (Laming, 1969).

Sequential effects of this sort obviously complicate the interpretation of choice RT data. They imply that the boundary conditions of a given experiment do not determine a single preparatory set that remains constant over trials. Rather, within the context of a fixed set of experimental conditions there can be several different states of preparation (i.e., several different combinations of perceptual and response bias), and the one that happens to prevail on any given trial is apparently determined in a rather complicated fashion by the local history of the stimulus sequence. To provide a complete account of a standard choice RT experiment then, two independent problems need to be solved: One must first identify the possible states of preparation and then be able to specify how transitions between these states are determined by the stimulus sequence. In the standard experiment these two problems must be studied and solved simultaneously.

From the standpoint of the usual motivation for studying choice RT, however, only the first of these problems is directly relevant; the second is essentially a problem in probability learning that happens to arise because of the random presentation schedules used in conventional experiments. From this point of view it would be more convenient to study RT biases in a setting where the bias on each trial is determined by the $E$, rather than by $S$ 's idiosyncratic response to the recent history of the stimulus sequence. The present study deals with an attempt to achieve this sort of control by beginning each trial (of an otherwise standard choice RT task) with a cue designating one of the choice stimuli. In the condition of primary interest, this cue indicated that the corresponding stimulus had a very high probability of being the one presented on that trial. Our hope was that in this case $S$ would be led to set himself for the cued stimulus without regard for events occurring on earlier trials.

The design of the cued RT task used here is as follows. Let $s_{1}, s_{2}, \cdots, s_{N}$ denote the choice stimuli; $c_{1}, c_{2}, \cdots, c_{N}$ the corresponding cues; and $c_{i n}$ and $s_{j n}$ the occurrence of $c_{i}$ and $s_{j}$ on Trial $n$. The trials are independent; $\gamma_{i}(i=1,2, \cdots, N)$ is the probability of Cue $c_{i}$

$$
\left(\sum_{i=1}^{N} \gamma_{i}=1\right)
$$

and $\pi_{i j}(j=1, \cdots, N)$ denotes the conditional probability of $s_{j n}$ given $c_{i n}$

$$
\left(\sum_{j=1}^{N} \pi_{i j}=1 \text { for each } i=1,2, \cdots, N\right) \text {. }
$$

The marginal probability of $\mathrm{s}_{\mathrm{j}}$

$$
\text { (i.e., } \left.\sum_{i=1}^{N} \gamma_{i} \pi_{i j}\right)
$$

is denoted by $\pi_{j}$. In all conditions of the present experiment, the cues were equally probable $\left(\gamma_{1}=\gamma_{2}=\cdots=\gamma_{N}\right)$, the cued stimulus $\left(s_{i}\right.$ for Cue $c_{i}$ ) was equally likely given any cue $\left(\pi_{11}=\pi_{22}=\cdots=\pi_{N N}\right)$, and each of the noncued stimuli was equally likely given any cue $\left(\pi_{\mathrm{ij}}=\right.$ constant for all $\mathrm{i}$ and $j$ when $i \neq j, j=1,2, \cdots, N)$. Consequently, the marginal stimulus probabilities were equal under all conditions, i.e., $\pi_{1}=\pi_{2}=\cdots=\pi_{\mathrm{N}}$.

The experiment reported below was designed to answer three interrelated questions about this cued RT task. First, does the cueing procedure eliminate (or at least substantially attenuate) sequential effects, in the sense that performance on any trial (at asymptote) is independent of previous events and depends only on the cue-stimulus combination on that trial? To examine this question, Ss were run under

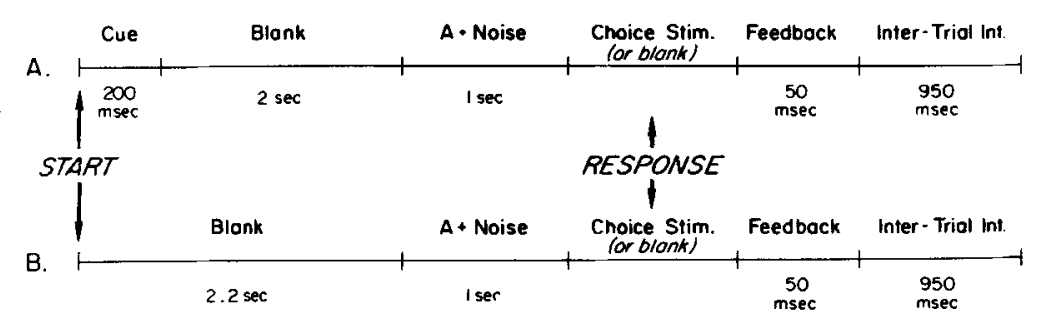


three conditions using the cueing procedure, and also in a control condition that involved the same RT task, but without the cues. If the cueing procedure is successful, sequential effects found in the control condition should be absent in the cue conditions-at least when $\pi_{\mathrm{ii}}$ is large. Second, the extent to which presentation of Cue $c_{i}$ biases $S$ in favor of the cued stimulus, $s_{i}$, will certainly depend on $\pi_{i i}$, i.e., on the validity of the cue. When $\pi_{i i}=1.0$, the bias effect will be large, since this amounts to a simple RT task with different stimuli on different trials. When $\pi_{\mathrm{ii}}=\pi_{\mathrm{ji}}=\pi_{\mathrm{i}}$, the cue conveys no information and we expect it to exercise relatively little control over S's bias. Between these extremes, the extent to which the cue determines the bias will depend on the difference between $\pi_{i i}$ and 1.0. To examine this dependence, three cue conditions were studied. In one (Condition ZP described below), the cue conveyed no information, in another (Condition PP) $\pi_{i i}=1.0$, and in the third condition (HP) $\pi_{i i}$ was much larger than $\pi_{i}$, but not equal to 1.0. On the basis of pilot work, there was some reason to believe that a cue with reasonably high validity would be about as effective as one with perfect validity; in this case, performance on the cued stimulus should be about the same in Conditions HP and PP.

Finally, if this last expectation is correct, the cueing procedure with $\pi_{i \mathrm{i}}<1$ allows us to study the effect of setting $S$ for one stimulus and then presenting another. In particular, it is possible to examine the extent to which biasing $S$ for the cued stimulus facilitates performance on other stimuli that have the same response assignment, or that share the same sensory modality. Facilitation of the first sort indicates a nonspecific response bias (LaBerge, Legrand, \& Hobbie, 1969); facilitation of the second kind would suggest an analogous "modality bias." In the present study there were four stimuli, two visual and two auditory, mapped onto two responses. One visual and one auditory stimulus were assigned to each response. With this arrangement, performance on a given stimulus could be compared as function of its relationship to the motor response and sensory modality indicated by the cue.

\section{Stimuli and Apparatus}

\section{METHOD}

Except for the cues, the design was that of a standard choice RT task with four choice stimuli and two responses. The choice stimuli were two colors, red and green, and two pure tones, "High" and "Low." Color stimuli were provided by an IEE Series 10 readout, which presented a luminous square of color ( $3 \times 3 \mathrm{~cm}, 5 \mathrm{ft}-\mathrm{L})$ surrounded by a dark background. The $S$ viewed this square at a distance of $40 \mathrm{~cm}$ through an oscilloscope viewing hood. The high $(1,250 \mathrm{~Hz})$ and low $(700 \mathrm{~Hz})$ tones were presented binaurally over earphones, each at $85 \mathrm{~dB}$ SPL. Both tone and color stimuli had a rise time from zero to peak intensity of approximately $25 \mathrm{msec}$ because the tones were gated through an electronic switch, and the onset of the colors involved some heating time of incandescent lamps in the readout. The earphones and IEE readout were also used to present additional stimuli that marked the foreperiod and provided information feedback, as described below.

Two circular response keys $(2.5 \mathrm{~cm}$ in diam) were located $15 \mathrm{~cm}$ apart on a sloping surface $30 \mathrm{~cm}$ below the viewing hood. The $S$ rested his left and right forefingers on these keys at all times, and responded by pressing the appropriate key. Each key had a vertical travel distance of $3 \mathrm{~mm}$ and required a pressure of $80 \mathrm{~g}$ to register a response.

For every $S$, the red square and high tone were assigned to the left-hand key, the green square and low tone to the right-hand key. Each session also included a small proportion of catch trials on which no choice stimulus was presented. The $S$ was instructed to make no response on these trials.

The Ss were run individually, se ated alone in a darkened room containing the stimulus display apparatus. A CDC-160 computer in an adjoining room controlled the event sequence and recorded responses.

Figure 1 shows the event sequence on each trial. In the cue conditions (Fig. 1a), each trial began with a cue, which consisted of a $200-\mathrm{msec}$ presentation of one of the four possible choice stimuli ( $S$ made no response at this point). After the cue there was a 2-sec blank interval, followed by a $1-\sec$ presentation of the letter A together with a 65-dB white noise. This was followed either by one of the four choice stimuli or by a blank interval in the case of catch trials. As soon as $S$ responded, information feedback on response speed was provided in the form of a $50-\mathrm{msec}$ presentation of a white square, together with white noise. This feedback event occurred only if $S$ 's response was faster than a certain fixed deadline that had been determined during pretraining. Following the feedback interval there was a 950-msec blank interval before the start of the next trial.
The event sequence was the same in the no-cue condition, except that the cue was omitted and replaced with a blank interval (Fig. 1b).

\section{Conditions}

There were four conditions, the no-cue (NC) condition, and three cue conditions that differed in the extent to which the cue predicted the stimulus: zero predictability (ZP), high predictability (HP), and perfect predictability (PP). Only one condition was in effect during any single session. Table 1 shows the event probabilities under each condition. Recall that $\gamma_{\mathrm{i}}$ is the marginal probability of Cue $c_{i}$, that $\pi_{i i}=P$ (Stimulus $s_{i} \mid$ Cue $\left.c_{i}\right)$, that $\pi_{i j}=P\left(s_{j} \mid c_{i}\right)$ where $\mathrm{j} \neq \mathrm{i}$, and that $\pi_{\mathrm{i}}$ is the unconditional (i.e., marginal) probability of $s_{i}$. In Table $1, i$ stands for any one of the four stimuli, i.e., $\pi_{\mathrm{ii}}=\mathbf{P}($ red stimulus $\mid$ red cue $)=\mathrm{P}($ low $\mid$ low $)$ $=\mathrm{P}($ green $\mid$ green $)=\mathrm{P}($ high $\mid$ high $) ; \pi_{i}=\mathrm{P}$ (red) $=P($ high $)=\ldots$, etc.; and $j$ stands for any stimulus other than $i$, so that $\pi_{i j}=P$ (red stimulus $\mid$ low cue $)=P($ high $\mid$ green $)=\ldots$, etc.

Each condition involved a certain proportion of catch trials as shown in the table. These occurred with equal probability following any cue. It should be noted that the probabilities $\pi_{i}, \pi_{i i}$, and $\pi_{i j}$ in Table 1 refer only to noncatch trials, so that, for example, $\pi_{\mathrm{ii}}$ is actually the conditional probability of $s_{i}$ given $c_{i}$ and given that the trial was not a catch trial. The various event probabilities for all trials (i.e., including catch trials) can be found by multiplying $\pi_{i}$, $\pi_{\mathrm{ii}}$, and $\pi_{\mathrm{ij}}$ by 1 minus the proportion of catch trials.

As Table 1 shows, Conditions NC and ZP were equivalent with respect to the amount of a priori information available to $S$ about the stimulus on any trial; in both cases, all four choice stimuli were equally probable on every trial. In Condition HP, on the other hand, the cued stimulus had a presentation probability eight times that of any noncued stimulus. And in Condition PP, noncued stimuli never occurred and the cued stimulus was always presented unless the trial was a catch trial.

Each session consisted of 10 blocks of trials, with the number of trials per block shown in Table 1 . The event probabilities given in the table held exactly within each block.

Table 1

Experimental Conditions

\begin{tabular}{|c|c|c|c|c|c|c|}
\hline Conditions & $\gamma_{i}$ & $\pi_{i}$ & $\pi_{\mathrm{ii}}$ & $\pi_{\mathbf{i j}}$ & $\begin{array}{c}\text { P(Catch } \\
\text { Trials) }\end{array}$ & $\begin{array}{l}\text { Trials } \\
\text { per } \\
\text { Block }\end{array}$ \\
\hline $\begin{array}{l}\mathbf{N C} \\
\mathbf{Z P} \\
\mathbf{H P} \\
\mathbf{P P}\end{array}$ & $\begin{array}{l}0 \\
.25 \\
.25 \\
.25\end{array}$ & $\begin{array}{l}.25 \\
.25 \\
.25 \\
.25\end{array}$ & $\begin{array}{l}.25 \\
.73 \\
1.0\end{array}$ & $0^{.09}$ & $\begin{array}{l}.14 \\
.14 \\
.15 \\
.15\end{array}$ & $\begin{array}{l}56 \\
56 \\
52 \\
52\end{array}$ \\
\hline
\end{tabular}




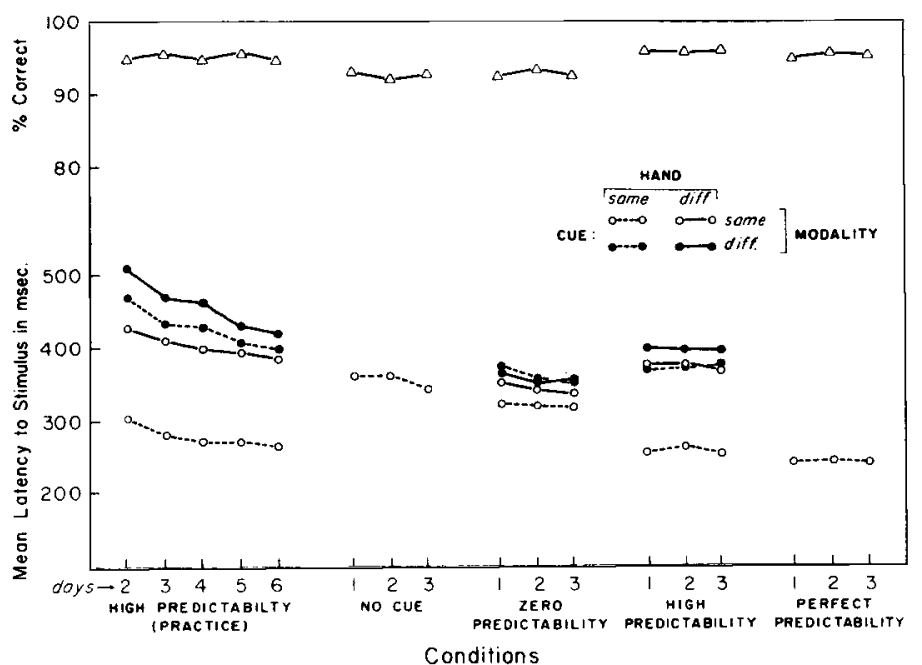

Subjects and Procedure

The Ss were four undergraduate volunteers. Each $S$ participated in 18 sessions, 1 session per day. The first day was devoted to training on various aspects of the RT task. Following this, each $S$ practiced for 5 consecutive days on Condition HP. During these sessions, Ss were told that the cue would indicate which stimulus was "most likely" to follow on that trial. Beginning with the seventh session, each $S$ ran for 3 consecutive days on each of the four conditions. The order of conditions was balanced across Ss by a Latin square design. Before starting a new condition, Ss were instructed as to the prevailing cue-stimulus contingencies.

The Ss were told to respond as fast as possible, but cautioned against making errors. During Days 2-6, a response speed deadline was established for each $S$ so that at least $60 \%$ of all responses were faster than this deadline with no more than $4 \%$ errors. This deadline then remained in effect during Days 7-18. The deadlines for the four Ss were $350,375,375$, and $400 \mathrm{msec}$. The Ss were paid a base rate of 754 per day plus 14 for each per cent of responses faster than the deadline, minus $2 ₫$ for each per cent of incorrect responses and minus $4 t$ for each response made on a catch trial.

\section{RESULTS}

Mean latencies and per cent errors from stimuli presented on Days 2 through 18 are shown in Fig. 2. Mean latencies to the four different stimuli under each condition are pooled, and the curve labels refer to the type of cue that was presented prior to the stimulus on a trial. For example, the lowest curves of the $\mathrm{ZP}$ and $\mathrm{HP}$ conditions represent the mean latencies given to Stimulus $s_{i}$ when the cue was $c_{i}$, averaged across all four stimuli. The upper three

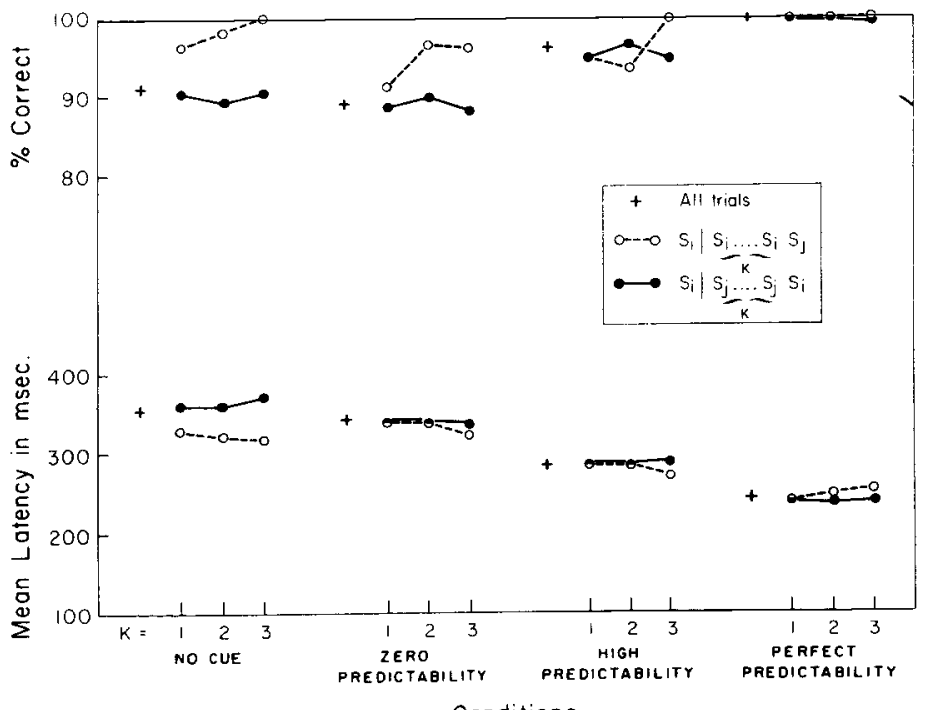

Conditions

Fig. 2. Mean latency and per cent correct response as a function of cueing conditions and days. Each point of a latency curve represents the mean latency to a stimulus given the type of cue that began the trial (except for the NC condition).

curves in these two conditions represent the mean latency given to Stimulus $s_{i}$ when the cue was $c_{j}(i \neq j)$. Each curve corresponds to a different relationship between cue and stimulus, as indicated by the legend. Thus, for example, the highest curve in the HP condition represents mean latency on all trials in which the presented stimulus differed from the cue stimulus in both its sensory modality and its response assignment, e.g., the stimulus was red (left-hand response) and the cue was a low tone (right-hand response).

Points on the lower curve of the HP condition are based on approximately 1,270 observations. Points of the upper curves of that condition are based on approximately 135 cases. Each of the points of the four $Z P$ curves is based on approximately 430 cases, and each of the points of the NC and PP conditions is based on approximately 1,750 cases. Per cent correct curves take in to account both catch and noncatch trials.

The data in Fig. 2 are based on averages across four Ss, four different stimuli, and both responses, but the pattern shown here does not differ in any significant respect from that shown by each $S$ on each stimulus-cue combination. In particular, the pattern of results for visual stimuli and auditory stimuli we re quite similar.

The main evidence relating to the effectiveness of the cue as a biasing agent is shown in the HP condition. Comparing latencies to a stimulus when it was the same as the cue vs when it was different yielded a difference of more than $100 \mathrm{msec}$. (No more than $8 \mathrm{msec}$ of this difference was due to the faster rise time of stimulus lamps upon close successive use, and repeated tones had no faster onsets than nonrepeated tones.) The significance of this amount of bias can be evaluated by comparing the lower curves across the four principal conditions of the experiment. The latency of Stimulus $s_{i}$ given Cue $c_{i}$ drops systematically as one moves from the NC condition across the graph to the PP condition, with the greatest drop occurring at the HP condition. The fact that the lower curve of the HP condition differs

Fig. 3. Mean latency and per cent correct response as a function of patterns of repetitions and nonrepetitions of stimuli. The open circles represent mean latency to Stimulus $s_{i}$ given exactly $k$ preceding trials of $s_{i}$. The filled circles represent mean latency to Stimulus $s_{i}$ given exactly $k$ preceding trials of $s_{j}\left(s_{i} \neq s_{j}\right)$. 


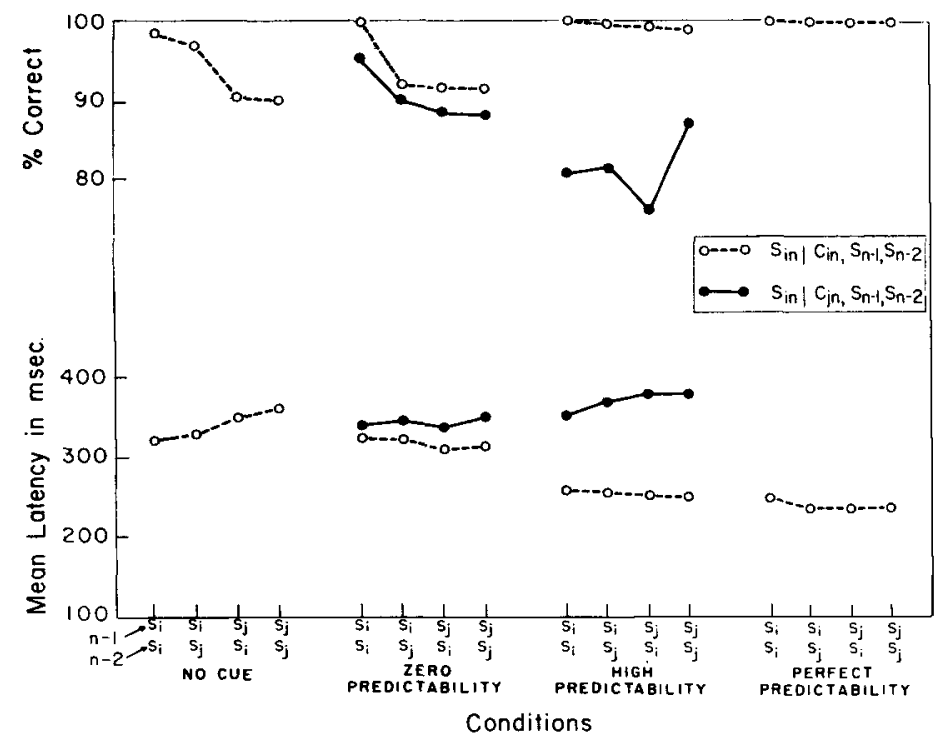

only slightly from the curve of the PP condition suggests strongly that the amount of bias produced by the HP condition was nearly maximum within the conditions of this experiment, even though $P\left(s_{i} \mid c_{i}\right)$ was only .73 .

Under the ZP condition, where a cue was given but carried no information about the stimulus to follow, there is a small but clear indication of a bias effect in favor of the cued stimulus. It is noteworthy here that the latencies to Stimulus $s_{i}$ given $\mathrm{Cue}_{\mathrm{j}}$ lie at a level above the control curve of the NC condition. And in the HP condition, these latencies are even higher than in the ZP condition. Thus the bias measure, being one of latency difference, reflects not only a lowering of the latency to Stimulus $s_{i}$ given Cue $c_{i}$, but also a heightening of the latency to Stimulus $s_{i}$ given Cue $c_{j}$.

An indication of the kinds of biases present can be gained from a closer examination of the levels of the four curves in the HP condition of Fig. 2. The test for response bias involves a comparison of latencies across hands. The appropriate comparison in the present design considers the trials in which the cue indicated a modality different from that of the stimulus that occurred, i.e., trials corresponding to the curves with filled circles in Fig. 2. When the cue was for a different modality but for the same hand as the presented stimulus, the latency was about $25 \mathrm{msec}$ faster than when the cue indicated both a different modality and a different hand.

The measure of perceptual bias requires comparisons of latencies to stimuli assigned to the same hand. In this design, there are two appropriate comparisons, one for each hand. When the comparison is made between cues indicating a different hand than the stimulus, but either the same or a different sensory modality, one obtains a measure of modality bias. The difference shown in Fig. 2 is approximately $25 \mathrm{msec}$. The same type of comparison on the hand that had the cued stimulus should give an estimate of a modality bias combined with a bias for a specific stimulus. The difference shown here is about $110 \mathrm{msec}$ for the combined bias. Subtracting from this value the modality bias estimated from the other hand yields a value of approximately $85 \mathrm{msec}$ as an estimate of the stimulus bias.

Analyses of sequential effects were based on trials between catch trials. The effects in the four conditions were first analyzed without regard to cue occurrences. Figure 3 shows the latency and frequency data. These are plotted as a function of exact run length, with runs of the same stimulus compared to runs of different stimuli. The latency points are for correct responses only. The number of observations for the repetition curve starts with over 5,000 cases per point at $k=1$, and decreases to 30 cases for $k=3$. The number of cases for nonrepetition curve points decreases only to about 1,200 cases.

The sequential data of the NC condition in Fig. 3 provide an appropriate baseline against which to judge the sequential analyses carried out on the other three conditions, where a cue occurred on each trial. In the $\mathrm{NC}$ condition, the repetitionand nonrepetition curves show slightly opposing slopes, with the repetition curves showing the faster latencies by about $30 \mathrm{msec}$ for one repetition, and increasing somewhat for two and three repetitions. The sequential latency data from the other three conditions show much attenuated sequential effects. In particular, the differences between repetitions and nonrepetitions of Run Lengths 1 and 2 virtually disappear for the ZP and HP groups.
Fig. 4. Mean latency and per cent correct response as a function of cueing conditions and patterns of same and different stimuli up to two preceding trials.

A second way of analyzing the sequential latency data took into account the cue that was presented on Trial $\mathrm{n}$. The results are shown in Fig. 4. For the NC condition, which serves as the main control in this analysis, the changes in latency due to the preceding two stimuli are substantial. Inspection of the latency curves of $s_{i} \mid c_{i}$ in the ZP, HP, and PP conditions shows a clear flattening effect compared to the $\mathrm{NC}$ condition.

Two other analyses of sequential effects were aimed at determining the possible presence of aftereffects of incorrect responses and catch trials on subsequent responding. These analyses are summarized in Figs. 5 and 6 . The data from the PP condition were omitted from Fig. 5 because only a very few observations were available for each point. The latency curves of Figs. 5 and 6 are all very nearly flat across six trials following an incorrect response or a catch trial. If there are any significant deviations from zero slope, they would appear to be negligibly small.

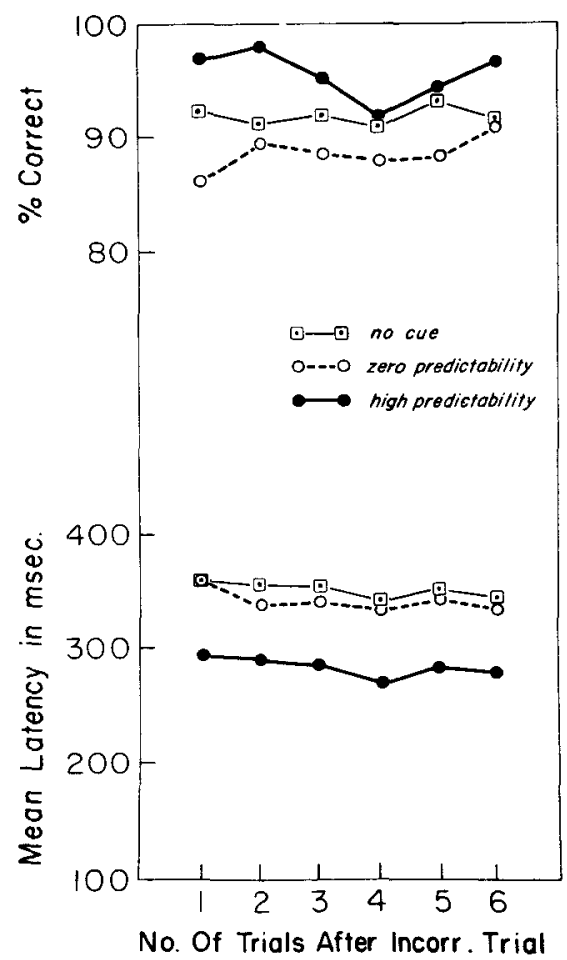

Fig. 5. Mean latency and per cent correct response as a function of ordinal trial number following an incorrect response with cueing condition as the parameter. 
Table 2

Mean Latencies in Msec of Correct and Incorrect (in Parentheses) Responses in Condition HP as a Function of the Modality and Hand Indicated by the Cue. Per Cent Correct Responses are Given Below the Latencies.

\begin{tabular}{ccc}
\hline & \multicolumn{2}{c}{ Cued Hand } \\
\cline { 2 - 3 } & Same as & Different from \\
& Stimulus & Stimulus \\
\hline Same as Stimulus & $256(308)$ & $373(227)$ \\
Cued Modality & $99.4 \%$ & $82.9 \%$ \\
Different from Stimulus & $372(351)$ & $398(318)$ \\
\hline
\end{tabular}

Finally, the breakdown of latencies from correct and incorrect responses in the HP condition as a function of the cue-stimulus combination are presented in Table 2 . Also included are the corresponding percentages of correct responses. Error latencies are quite a bit faster than correct latencies when the cue is for a stimulus on a different hand, and, if anything, only slightly faster when the cue is for the same hand but for a different stimulus. However, error latencies are moderately greater than correct latencies when the cue is the same as the stimulus. This last comparison is based on a total of only 20 errors, but the direction of the difference was the same for each of the four Ss.

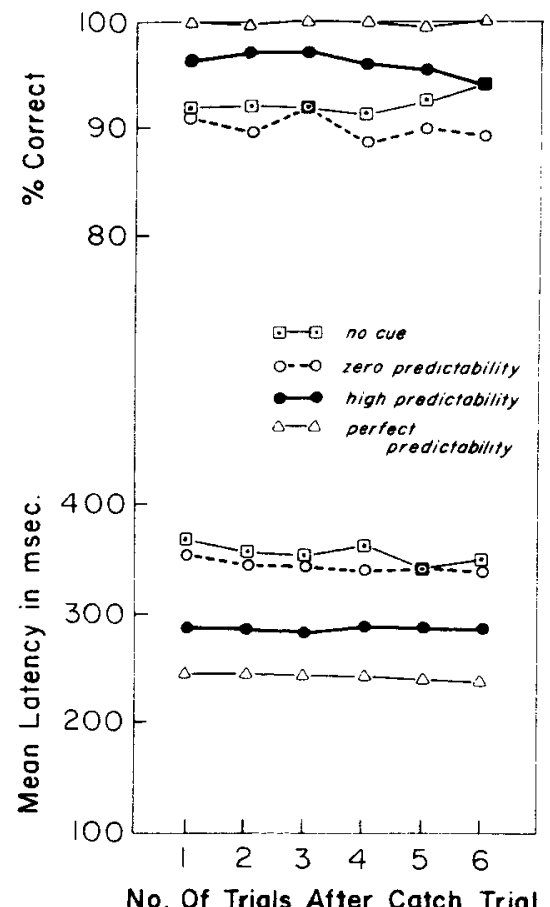

Fig. 6. Mean latency and per cent correct response as a function of ordinal trial number following a catch trial, with cueing condition as the parameter. where $\pi_{\mathrm{ii}}=1$. simple RT task. alternatives would, with better-than-chance probability, form a bias toward the one that had just appeared as a cue. An interesting control condition would be one in which the cues are symbolic (e.g., names of the stimuli) rather than actual physical samples of the stimuli. This would eliminate that part of the stimulus bias due to "matching to sample" behavior, and perhaps eliminate bias effects in the ZP condition. But even if the obtained bias were smaller, it is not likely that the curves would lie outside the range bounded by the curves of the $\mathrm{ZP}$ condition, since omission of the cue entirely in the NC condition produced a curve within that range.

Turning to the sequential analyses, it In the conditions of the experiment where a cue was presented on a trial, response times were consistently and often substantially faster to a stimulus $s_{i}$ given Cue $c_{i}$ than to Stimulus $s_{j}$ given $c_{j}$. This constitutes clear evidence that the present cueing technique produces a bias in this RT task. That the technique is a very effective means of controlling much of the available bias in this situation, even when $\pi_{\mathrm{ij}}$ is as low as .73 , is evident from the fact that the lowest curve of the high predictability condition approximates very nearly the curve of the perfect predictability condition

The level attained under the PP condition quite probably is not a minimum even though the condition closely resembles the simple RT task. The reason for this is that in this condition (as was the case in all the other conditions), catch trials were presented about $15 \%$ of the time. Very probably, omission of these catch trials or lowering the penalty for responding to them would have produced faster responses in this condition. One other factor that may have attenuated the degree of response bias possible in this condition is the fact that the responses were distributed randomly between the two hands. This could well prevent the sort of accumulation of muscle tension that may occur when only one hand is used to make the responses, as in the

Considering the results of the ZP condition, where the cue carried no information about the stimulus, it seems at first odd that a bias effect appeared, even though it was a relatively small one. However, given the large amount of training (in Days 2-6) with a cue that carried helpful information, it is perhaps not surprising to find that the bias function of the cue transferred to a neutral condition such as the ZP case. The small suggestion of convergence of the latency curves over the 3-day period of this condition is consistent with this reasoning. But even without a transfer effect, one might expect that a $S$ confronted with four equally likely stimulus appears that the presentation of a cue sharply reduces sequential latency effects of the repetition and nonrepetition variety while it is inducing a bias within a trial. There is no direct way to tell whether or not other, more complex, sequential stimulus patterns could still be exerting effects that cancel each other when averaged over trials. However, this possibility seems somewhat remote in view of the large amount of bias being taken up already by the cueing in the $\mathrm{HP}$ condition.

The presence of sequential effects in the dependent variables of most choice experiments has been routinely accepted as part of the experimental data. In fact, these phenomena have been so pervasive that effects to be basic phenomena in themselves, and models have been formulated for the purpose of accounting for them (e.g., Falmange, 1965). An alternative approach is to control these effects in such a way as to discover experimentally what processes underlie them. One way to do this is to build into the series of trials specific recurring sequential patterns and then to note the effects on choice data. Unfortunately, there is no assurance that the $S$ will perceive the patterns in the way intended by the $E$. This means that the effective stimulus pattern is under the control of the $S$, rather than the $E$.

The approach motivating the present technique considers sequential dependencies to be a nuisance, and strives to eliminate them altogether. This does not mean that the attentional or biasing processes commonly assumed to underlie sequential effects are to be eliminated as well. Rather, the effort is directed toward transferring the control of these processes away from sequential patterns and placing them instead under the control of an experimentally manipulatable event within the trial itself. In this way, the S should be able to approximate more closely a steady state for presentations of the same stimulus over a series of trials, with the result that effects of other independent variables can be some investigators consider the sequential 
more clearly revealed. One principal finding of the present study highlights this point: In Fig. 2, the curves of the HP condition clearly reveal modality effects that are completely concealed in the curve of the NC condition. When a cue is given, and the $E$ has reasonable certainty that the $S$ is biased for that specific stimulus and modality, then, if a stimulus of another modality is presented, he can estimate the time it takes to shift away from the biased modality and process a stimulus of a different modality.

\section{REFERENCES}

BERTELSON, P. Sequential redundancy and speed in a serial two-choice responding task.
Quarterly Journal of Experimental Psychology, 1961, 13, 90-102

BERTELSON, P. S-R relationships and reaction times to new versus repeated signals in a serial task. Journal of Experimental Psychology, 1963, 65, 478-484.

FALMANGE, J. C. Stochastic models for choice reaction time with applications to experimental results. Journal of Mathematical Psychology, $1965,2,77-124$

LaBERGE, D., LEGRAND, R., \& HOBBIE, R. K. Functional identification of perceptual and response biases in choice reaction time. Joumal of Experimental Psychology, 1969, 79, 295-299.

LAMING, D. R. J. Subjective probability in choice-reaction experiments. Journal of Mathematical Psychology, 1969, 6, 81-120.

WILLIAMS, J. A. Sequential effects in disjunctive reaction time: Implications for decision models.
Journal of Experimental Psychology, 1966, 71, 665-672.

\section{NOTES}

1. This research was supported by Public Health Service Research Grant No. MH 16270-01 and the Center for Research in Human Learning (National Science Foundation Grant GS-541). We thank Betty Hamre, who assisted in running the experiment.

2. Address: Department of Psychology, University of Minnesota, Minneapolis, Minnesota 55455 .

(Accepted for publication May 9, 1969.) 